\title{
Mobility and fill factor correlation in geminate recombination limited solar cells
}

\author{
Mattias Andersson, Christian Mueller, Bekele H Badada, Fengling Zhang, \\ Uli Wuerful and Olle Inganäs
}

\section{Linköping University Post Print}

N.B.: When citing this work, cite the original article.

Original Publication:

Mattias Andersson, Christian Mueller, Bekele H Badada, Fengling Zhang, Uli Wuerful and Olle Inganäs, Mobility and fill factor correlation in geminate recombination limited solar cells, 2011, Journal of Applied Physics, (110), 2, 024509.

http://dx.doi.org/10.1063/1.3609079

Copyright: American Institute of Physics (AIP) http://www.aip.org/

Postprint available at: Linköping University Electronic Press

http://urn.kb.se/resolve?urn=urn:nbn:se:liu:diva-70757 


\title{
Mobility and fill factor correlation in geminate recombination limited solar cells
}

\author{
L. Mattias Andersson, ${ }^{1, a)}$ Christian Müller, ${ }^{2}$ Bekele H. Badada, ${ }^{3}$ Fengling Zhang, ${ }^{1}$ \\ Uli Würfel, ${ }^{4,5}$ and Olle Inganäs ${ }^{1}$ \\ ${ }^{1}$ Biomolecular and Organic Electronics, Department of Physics, Chemistry and Biology, Linköping University, \\ SE-581 83, Linköping, Sweden \\ ${ }^{2}$ Institut de Ciència de Materials de Barcelona (ICMAB-CSIC), Esfera UAB, ES-08193 Bellaterra, Spain \\ ${ }^{3}$ Department of Physics, University of Cincinnati, Cincinnati, Ohio 45221-0011, USA \\ ${ }^{4}$ Fraunhofer Institute for Solar Energy Systems ISE, Heidenhofstr. 2, 79110 Freiburg, Germany \\ ${ }^{5}$ Freiburg Materials Research Centre FMF, Stefan-Meier-Str. 21, 79104 Freiburg, Germany
}

(Received 30 March 2011; accepted 6 June 2011; published online 26 July 2011)

\begin{abstract}
Empirical data for the fill factor as a function of charge carrier mobility for two different polymer:fullerene systems is presented and analyzed. The results indicate that charge extraction depth limitations and space charge effects are inconsistent with the observed behavior, and the decrease in the fill factor is, instead, attributed to the field-dependent charge separation and geminate recombination. A solar cell photocurrent limited by the Onsager-Braun charge transfer exciton dissociation is shown to be able to accommodate the experimental observations. Charge dissociation limited solar cells always benefit from increased mobilities, and the negative contribution from the reduced charge separation is shown to be much more important for the fill factor in these material systems than any adverse effects from charge carrier extraction depth limitations or space charge effects due to unbalanced mobilities. The logarithmic dependence of the fill factor on the mobility for such a process is also shown to imply that simply increasing the mobilities is an impractical way to reach very high fill factors under these conditions since unrealistically high mobilities are required. A more controlled morphology is, instead, argued to be necessary for high performance. (C) 2011 American Institute of Physics. [doi:10.1063/1.3609079]
\end{abstract}

\section{INTRODUCTION}

\section{A. Background}

It is a well-established fact that the fill factor (FF) of solar cells correlates with the mobility of charge carriers. In the case of organic solar cells, several studies report an improvement in the FF with increasing mobilities, ${ }^{1-3}$ however, these variations are usually concomitant with other significant variations, e.g., of the morphology. In addition, both the hole and electron mobilities usually change simultaneously, which obscures the fundamental relationship between the mobility and FF. Another aspect to consider is that there are plenty of device-related parameters, such as the active layer thickness as well as shunt- and series- resistances ${ }^{4}$ that influence the FF of a solar cell. Thus, the data in the literature can vary very strongly for a given material system. The fundamental question that will be investigated here is how the FF is influenced by mobility. As we will show, answering this question also yields information about the physics of the device operation.

Although the correlation between mobility and FF may seem obvious and well understood based on the body of work available for inorganic semiconductors, there are some aspects that are specific to organic materials, most of which are related to the excitonic nature of the latter and their comparatively small valence and conduction bands. The strongly

\footnotetext{
a) Author to whom correspondence should be addressed. Electronic mail: matan@ifm.liu.se.
}

bound excitons of organic materials is the reason why twophase systems are employed. However, even in many twophase systems charge separation is strongly dependent on both the mobility and applied electric field. ${ }^{5}$ This, in turn, can be reflected in the FF. Furthermore, the small site density of the valence and conduction bands leads to localization and activated charge transport, which is different from the ballistic charge transport of common inorganic crystals which adds to the complexity. One example of this is that charge carriers are not created at thermal equilibrium. They will exhibit a time-varying mobility during a significant portion of the extraction process, ${ }^{6}$ which may also influence the FF. The highest theoretical efficiencies ${ }^{7}$ in polymer:fullerene solar cells are obtained with materials combinations and in device geometries that operate at the very limit of charge dissociation and extraction, where the impact from these organic specific properties on the FF is the largest. The relationship between the FF and mobility in organic solar cells can thus be different from that of inorganic materials such as silicon, and it is also different for different combinations of organic materials.

The performance limiting processes, which involves the movement of charge carriers and thus may affect the FF, can be divided in two categories; (1) the separation of charge carriers, and (2) the transport of charge carriers to the electrodes. After excitation, the charge carriers must be separated before geminate recombination occurs. This process is dependent upon, among other factors, charge carrier mobility and applied field. ${ }^{8}$ As long as this process is operating at less 
than unity efficiency, the FF will be reduced since the current will be field dependent between open and short-circuit conditions. Higher mobilities reduce the necessary electric field and increase the FF.

The material systems that will be reported on here are the two polymers poly[2,7-(9,9-dioctylfluorene)-alt-5,5-(4,7di-2-thienyl-2,1,3-benzothiadiazole)] (APFO-3) ${ }^{9}$ and poly[2,7(9,9-dioctylfluorene)-alt-5,5-(5,7-di-2-thienyl-2,3-bis(4-(2-ethylhexyloxy)phenyl)thieno[3,4-b]pyrazine)] (APFO-Green 5), ${ }^{10}$ in blends with the fullerene [6,6]-phenyl- $\mathrm{C}_{61}$-butyric acid methyl ester ([60]PCBM). Experimental evidence is presented that permits the following conclusions for solar cells based on these and similar materials, which are different from the conventional views based on transport commonly relied upon:

(1) the FF is not limited by the actual transport of charge carriers to the electrodes and bimolecular recombination;

(2) the FF is limited by carrier dissociation and geminate recombination;

(3) as a result, there is no benefit from balanced mobilities in this regime.

These conclusions are consistent with other reports that have demonstrated geminate recombination to be the performance-limiting mechanism in these materials. ${ }^{11}$ In most organic donor/acceptor systems the geminate processes should be present to some degree and will have to be considered, relative to other types of recombination.

\section{B. Charge generation}

In polymer/fullerene solar cells, charges are formed at the interface between the polymer donor and the fullerene acceptor. The sequential processes en route to power generation are; optical absorption, exciton diffusion, the splitting of charges at donor/acceptor interfaces, geminate recombination of these charges at early times, formation of free polarons, charge transport in the separate domains of hole and electron transport, recombination and trapping at longer times with bimolecular or monomolecular kinetics, and charge extraction at the interface to the electrodes. The mechanism of the formation and stabilization of free charge carriers has received considerable attention ${ }^{12,13}$ but the details remain controversial. There is evidence that the formation of charge carriers occurs via a charge transfer state, which also determines the photovoltage of the devices. ${ }^{14} \mathrm{In}$ recent experiments the behavior of photo-generated charges has been followed by transient absorption in devices with an applied field for two different polymer/fullerene systems, with widely different results and interpretations. ${ }^{15,16}$ The transient absorption studies rely on the spectroscopic assignment of optical absorption to charged species, but cannot identify those that are eventually collected as the photocurrent. Charge transfer recombination is more visible in the photoluminescence from this state, which is in direct competition with the generation of the photocurrent. Studies of the steady-state field dependence of photoluminescence quenching and photocurrent generation in some low bandgap polymer/fullerene systems ${ }^{17-19}$ yield results indicating that the collection of charge carriers and the suppression of photolu- minescence do not always have the same field dependence, arguing that there are contributions from both geminate and bimolecular recombination in such systems or that there could be more than one route of photocurrent generation.

Relevant photo-physical processes operate at different timescales and the overlap of the optical and the electrical transient windows is not adequate to produce a complete picture. Tracking the steady-state behavior with transient-type measurements also raises considerable difficulties, and distinguishing the contributions from charge separation, recombination, and transport in the presence of an electric field is a delicate task. Nevertheless, transient absorption measurements on alternating polyfluorene (APFO)-class polymers ${ }^{11}$ have shown that the dominant recombinative loss mechanism in those materials is of a geminate nature, i.e., the loss in current occurs before the charge carriers are truly separated. Thus, it is reasonable to expect that the FF is not primarily limited by Schubweg or space charge effects but rather by the field dependence of charge separation.

\section{Mobility manipulation and experimental system}

The electron and hole mobilities of a given polymer:fullerene system can be changed in several ways. Some of the more common engineering approaches to performance enhancement are to vary the stoichiometry of the active layer blend, the molecular weight of the polymer, or to change the blend morphology by annealing. However, besides charge transport this usually also influences other aspects relevant for device performance, e.g., light absorption. Charge generation and recombination are also influenced by other factors, e.g., the shifting of energy levels during crystallization, such as for poly(3-hexylthiophene) (P3HT). Annealing P3HT:[60]PCBM blends has been shown to drastically increase solar cell performance. This enhancement coincides with an increase in mobility, ${ }^{20}$ however, it has also been linked to increased absorption and to morphology. Although both electron and hole mobilities simultaneously vary, and the data is not presented explicitly as FF versus mobility, there seems to be a roughly linear correlation between FF and $\log (\mu)$ over more than 3 orders of magnitude in mobility in the data of Figs. 3 and 6 in Ref. 20, for different annealing temperatures of P3HT:[60]PCBM blends. This is at variance with a purely transport-limited case, for which less than 2 orders of magnitude in mobility increase from the point where the FF starts to increase would be sufficient to saturate the FF close to the non-transport-limited value. Here, it will be demonstrated that such a correlation can be explained by a geminate recombination.

The APFO-class polymers are typically liquid-crystalline, but do not crystallize. Several of these polymers have similar solubility parameters ${ }^{21}$ along with [60]PCBM, and [60]PCBM is thus soluble in the polymer phase to some extent, especially if a low molecular weight polymer grade is used. Photoluminescence (PL) studies show that 20\% [60]PCBM is sufficient to effectively quench polymer emission, ${ }^{22}$ and transient absorption measurements show only minor differences in geminate recombination times between different stoichiometries. ${ }^{11}$ The polymer is, by far, the 
strongest absorber at most of the visible wavelengths even at very high levels of [60]PCBM loading in these binary blends. Thus, there is a wide range of stoichiometries that permit production of films that differ mostly in their charge carrier transport properties. Regarding the other important processes connected to solar cell operation, i.e., the optical absorption event and the geminate recombination time, the films are highly comparable. Here, it is assumed that any charge generated from the [60]PCBM excitations is negligible, which is in good agreement with the experimental data. As long as there is percolation of the polymer phase, ${ }^{23,24}$ the hole transport is relatively independent of the stoichiometry. This observation has previously been reported for blends comprising [60]PCBM and either APFO-3 or APFO-Green 5. Hence, for a given, and sufficiently high, molecular weight, these blends display a constant hole mobility but strongly varying electron mobilities for different PLquenched, i.e., [60]PCBM-rich, stoichiometries where the properties that depend on the proximity of [60]PCBM to the polymer have largely saturated. In general, the mobilities appear to correlate well with the number and difficulty of intermolecular charge carrier jumps. For the small molecule [60]PCBM, the electron mobility is proportional to the volume fraction, while in the polymer case high molecular weights can retain high mobilities even at very low volume fractions due to the inherent linking of transport sites. ${ }^{24}$ Furthermore, we have found that different polymer synthesis batches of APFO-3 tend to display dissimilar pure phase (and concomitantly blend phase) hole mobilities. Since the electron mobility only depends on the stoichiometry, it is thus also possible to vary the hole mobility while keeping the electron mobility constant. In this way, both mobilities can be independently varied in an otherwise specified system using stoichiometry and polymer molecular weight/batch differences. Of particular interest is the fact that the electron mobility is typically lower than the hole mobility in high molecular weight APFO-3-based blends, while the opposite (higher electron than hole mobility) holds for APFO-Green 5-based blends, so that the difference between electron and hole mobility can be made to either increase or decrease with the increase of the parametric mobility, depending upon the choice of system.

Much of the experimental data herein is compiled from previous publications and any additional measurements have been carried out according to previously reported procedures. Experimental information can be obtained from the appropriate references, ${ }^{9,10,24}$ which are also indicated in conjunction with the discussion of the respective data.

\section{RESULTS AND DISCUSSION}

\section{A. Theoretical calculations}

To put the experimental polymer:fullerene data into perspective vis-à-vis dissociation, Schubweg limitations, and space charge effects, zeroth order calculations and computer simulations that show the FF as a function of mobility are shown in Fig. 1. Within the Onsager-Braun framework, ${ }^{8}$ the dissociation rate constant $\left(\mathrm{k}_{\mathrm{d}}\right)$ is directly proportional to the mobility, and in the absence of later stage recombination, the
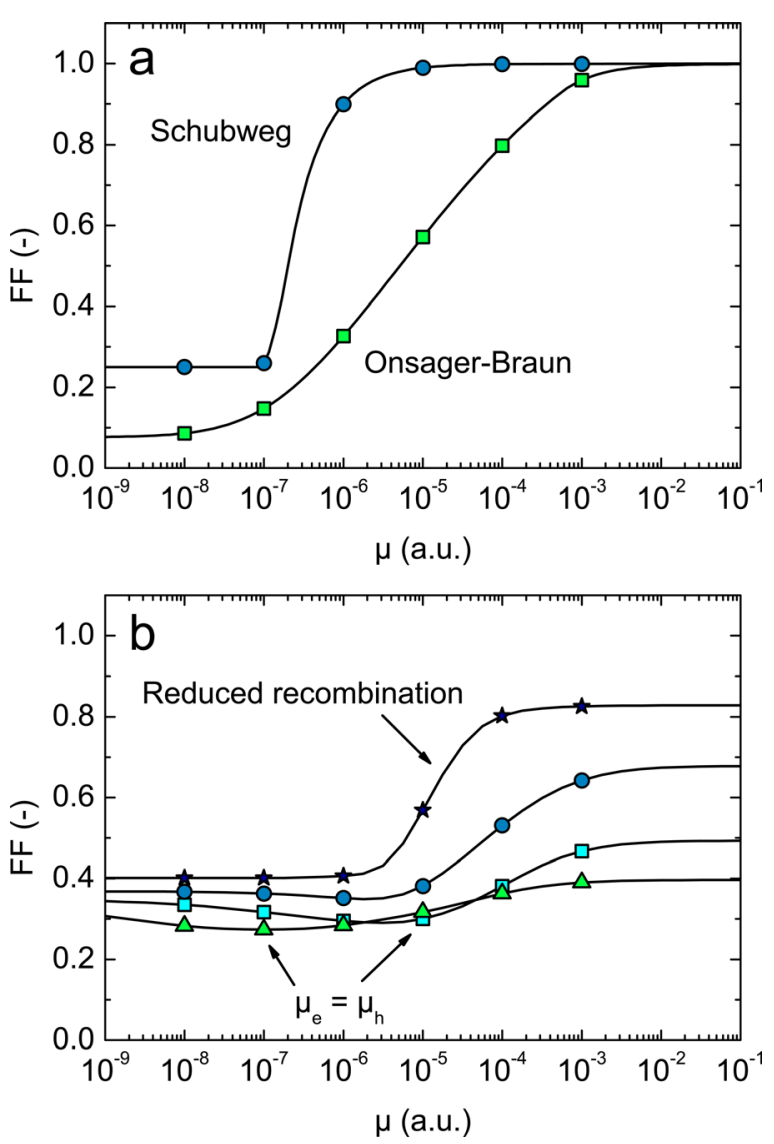

FIG. 1. (Color online) Zeroth-order calculations of the FF as a function of mobility for a dissociation-limited process (squares) and a transport-limited process (circles). (a) In the Onsager-Braun case, it is the sum of the mobilities that is the parameter and in the Schubweg case the mobilities are equal. (b) Numerical simulation results for three different fixed mobilities, $10^{-4}$ (circles), $10^{-5}$ (squares), and $10^{-7} \mathrm{~cm}^{2} \mathrm{~V}^{-1} \mathrm{~s}^{-1}$ (triangles), as well as for $10^{-4} \mathrm{~cm}^{2} \mathrm{~V}^{-1} \mathrm{~s}^{-1}$ with a recombination rate reduced by a factor of 100 (stars).

current of a solar cell is proportional to the dissociation probability (P). With $\mathrm{P}=\mathrm{k}_{\mathrm{d}} /\left(\mathrm{k}_{\mathrm{f}}+\mathrm{k}_{\mathrm{d}}\right)$, where $\mathrm{k}_{\mathrm{f}}$ is the recombination rate constant, and with material parameters representative for the materials used here, the FF will approach a fixed value of $\sim 0.1$ as $\mu \rightarrow 0$, increase rapidly around $\mathrm{k}_{\mathrm{f}} \sim \mathrm{k}_{\mathrm{d}}$, and approach 1 as $\mu \rightarrow \infty$. That the FF never goes to 0 is due to $\mathrm{P}$ always retaining its functional form for all non-zero mobilities. At $\mu=0, \mathrm{P}=0$ and $\mathrm{FF}$ is undefined. For the transport to the electrodes, two main mechanisms are influencing the FF; bimolecular recombination and space charge effects. It is possible to define the distance a carrier can travel before it recombines (Schubweg, $\mathrm{d}$ ), as $\mathrm{d}=\mu \tau \mathrm{E}$, where $\mu$ is the mobility, $\tau$ is the carrier lifetime, which, in the case of bimolecular recombination, is carrier density dependent, and $\mathrm{E}$ is the electric field. If the built-in field is approximated as, $E_{b i}=V_{o c} / D$, where $V_{o c}$ is the open circuit voltage and $\mathrm{D}$ is the device thickness, and with both mobilities equal, this will result in $\mathrm{FF}=0.25$ as long as $\mathrm{d}<\mathrm{D}$ at short- circuit. The FF will rapidly increase with the increasing $\mu \tau$-product over about 1 order of magnitude when $\mathrm{d} \geq \mathrm{D}$ at short-circuit, and then asymptotically approach unity as $\mathrm{d} \rightarrow \mathrm{D}$ at $\mathrm{V}_{\mathrm{oc}}$. If the $\mu \tau$-products of holes and electrons are unbalanced, either the extraction depth is proportional to 

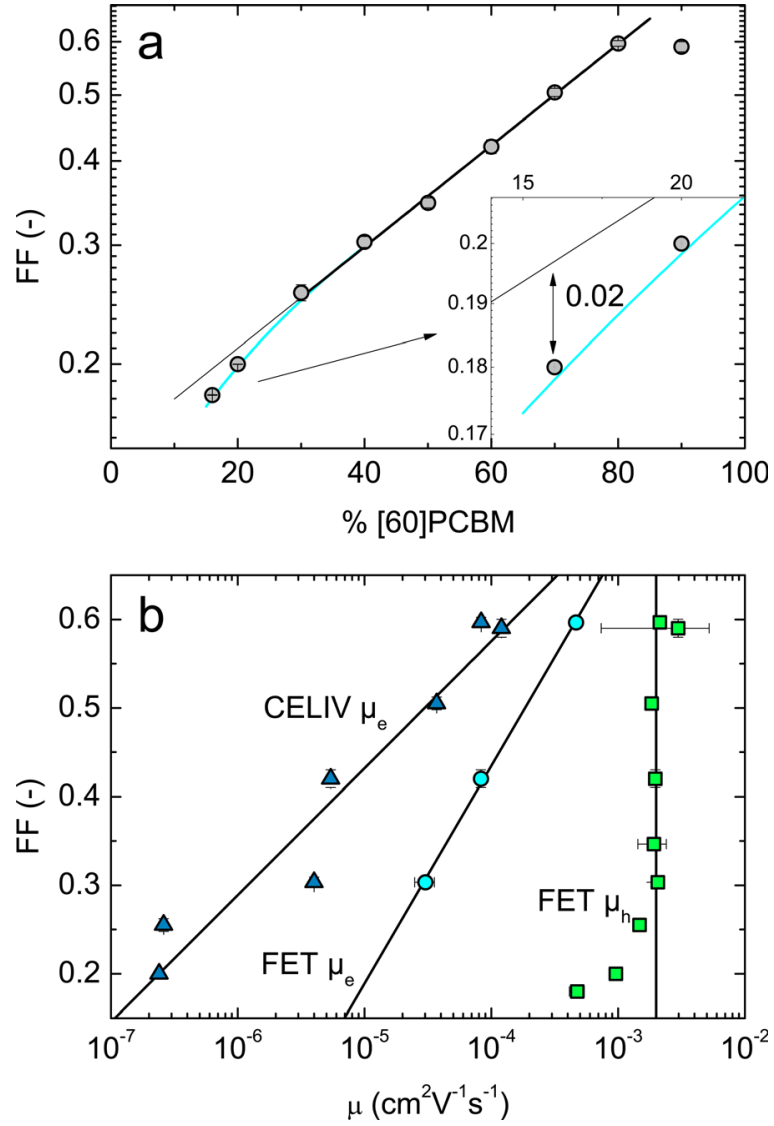

FIG. 2. (Color online) The FF as a function of (a) stoichiometry, and (b) mobility. The CELIV electron (triangles), and FET electron (circles) and hole (squares) mobilities for APFO-3:[60]PCBM are shown. The (FET) hole mobility is constant at $2 \times 10^{-3} \mathrm{~cm}^{2} \mathrm{~V}^{-1} \mathrm{~s}^{-1}$ over most of the FF range, while the FF increases linearly with $\log \left(\mu_{\mathrm{e}}\right)$.

$\mathrm{V}^{[1 / 2]}$, or there will be a buildup of space charge, both of which will limit the FF to 0.38 for $\mathrm{E}_{\mathrm{bi}}=\mathrm{V}_{\mathrm{oc}} / \mathrm{D}$ with the current, J, proportional to $\mathrm{V}^{[1 / 2]}{ }^{25}$ Dissociation-limited FF can thus be lower than the 0.25 or 0.38 minima of transport or space-charge-limited FF. Furthermore, the mobility range over which the FF increases is considerably larger for dissociation-limited systems.

In this case, the increase in FF is approximately linear with $\log (\mu)$ over 4 orders of magnitude around $\mathrm{k}_{\mathrm{d}}=\mathrm{k}_{\mathrm{f}}$ for the Onsager-Braun process, and in the Schubweg-limited case, the FF increases strongly with mobility only in a small interval after the point where $\mathrm{d}=\mathrm{D}$ at short- circuit conditions [Fig. 1(a)]. Mathematical expressions for the synthetic J-V curves used to evaluate the FF can be found in the Appendix. Figure 1(b) shows some representative numerical results for the FF as a function of mobility based on a full device model but solely with bimolecular recombination. For high parametric mobility values, the FF is almost constant and depends on the other mobility. For low parametric mobility values, the FF is limited by either space charge effects or extraction depth according to $\mathrm{J}$ proportional to $\mathrm{V}^{[1 / 2]}$, and at low but equal mobilities by Schubweg at $\mathrm{FF}=0.25$. It can clearly be seen that a reduction of the mobility has a severe impact on the FF, but the fundamental shape of all of the charge extraction limited calculations remains the same with a FF always above 0.25 and a steep increase in FF over a rather narrow mobility range of about 2 orders of magnitude. Detailed information about the numerical device model can be found in the Appendix.

\section{B. Experimental results}

Figures 2(a) and 2(b) show the FF of the APFO$3:[60] \mathrm{PCBM}$ blends versus the experimental parameters of stoichiometry and mobility, respectively. As expected, the field-effect transistor (FET) and charge extraction by linearly increasing voltage (CELIV) data behave in the same way, but there is a bigger scatter in the CELIV mobility data compared to the FET data. From Fig. 2(a), it is obvious that the scatter in FF is small and that the trend is rather featureless apart from the data points that correspond to polymer-rich samples, for which the hole mobility starts to deviate. The electron mobility of the APFO-3:[60]PCBM blends is difficult to extract from the FET measurements since the hole mobility is much higher than the electron mobility and the threshold voltages are such that the electron mobility is usually not possible to resolve. ${ }^{9}$ In the case of CELIV measurements the problem is the opposite; the hole mobility is so high that the CELIV hole extraction peak is obscured by the
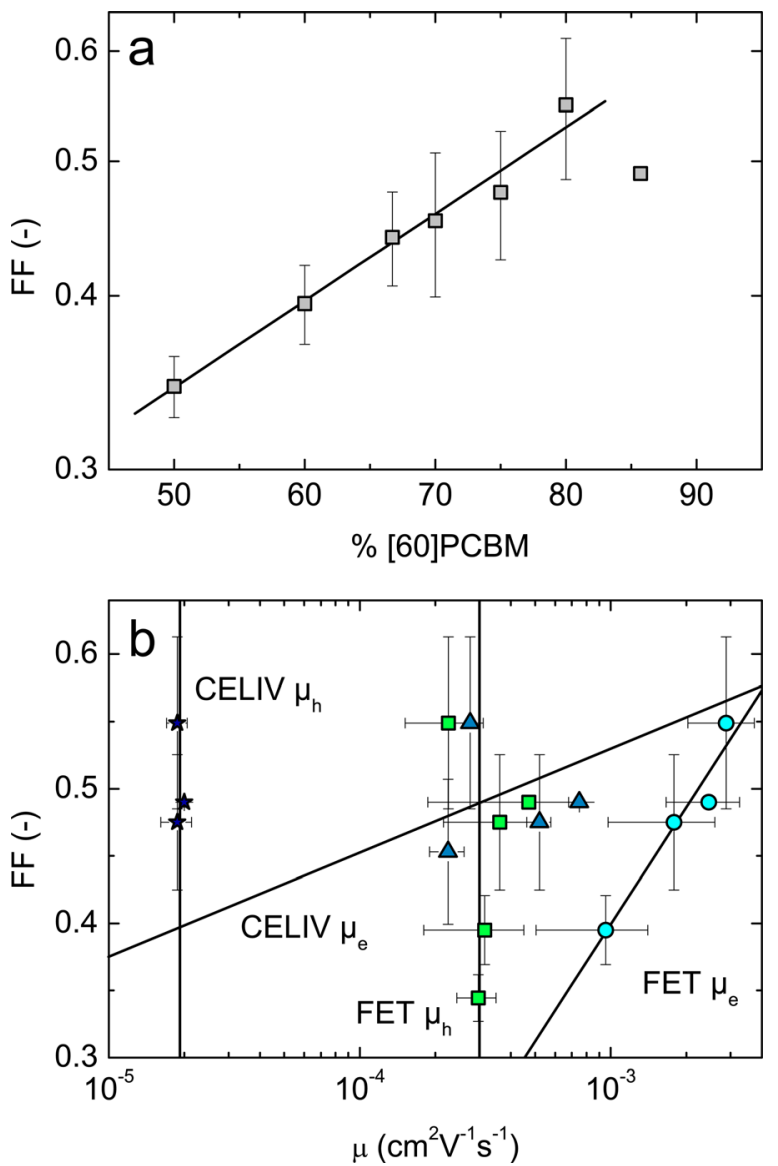

FIG. 3. (Color online) The FF as a function of (a) stoichiometry, and (b) mobility. The CELIV electron (triangles) and hole (stars), and the FET electron (circles) and hole (squares) mobilities for APFO-Green 5:[60]PCBM blends are shown. The CELIV and FET hole mobilities are constant at $2 \times 10^{-5}$ and $3 \times 10^{-4} \mathrm{~cm}^{2} \mathrm{~V}^{-1} \mathrm{~s}^{-1}$ respectively, while the FF increases linearly with $\log \left(\mu_{\mathrm{e}}\right)$. The error bars are comparatively large due to large sample populations with a considerable thickness variation and a less stable morphology than APFO-3. 
sample RC-constant for thin samples. Furthermore, the electron mobility is too low to observe an extraction peak during the time of the extraction ramp for the most polymer-rich samples. It is thus very difficult to obtain a complete set of mobilities for both measurement techniques, however, they complement each other well.

Here, the argument will be made that the data in Fig. 2 is inconsistent with a Schubweg or space-charge-limited mobility since the FF increase linearly over more than 2 orders of magnitude in mobility, there are no features in the FF versus stoichiometry data that can be identified with a transition between different limitations, and the FF follows a constant trend below a value of 0.25 . As is evident from Fig. 1, a Schubweg-limited process should not display a linear dependence of the FF on $\log (\mu)$ over such a wide mobility range and should never decrease below a FF of 0.25 . Furthermore, a Schubweg-based model for the behavior seen in Fig. 2 would require the electron mobility to vary in an unphysical way with respect to stoichiometry; a rapid increase at low loading level, slowly increasing for intermediate levels, and then again rapidly increasing at high levels, which is far from the observed behavior. Instead, a charge-separationbased model is a much better alternative. The increase in FF is about 0.25 units per decade of mobility, with only minor differences between the FET and CELIV data.

Figure 3 shows the FF of the APFO-Green 5:[60]PCBM blends as a function of the experimental parameter stoichiometry [Fig. 3(a)], and both FET and CELIV electron and hole mobilities [Fig. 3(b)]. In this case the available stoichiometry range, and thus electron mobility range, is smaller due to material availability, but the FF data are based on average values collected from about 100 solar cells. The mobility data is based on a smaller sample population. In particular, the CELIV electron mobilities are evaluated at conditions near where the RC effects are important, and it is possible that the higher capacitances due to slightly lower sample thicknesses, together with the higher mobility of the 1:4 samples, are responsible for an underestimate of the electron mobility there. Again, the hole mobility is rather constant, however, in this case it is always lower than the electron mobility, as opposed to the APFO-3:[60]PCBM case where it is always higher. Just as for the APFO$3:[60] \mathrm{PCBM}$, the increase in FF is still on the order of 0.25 units per decade of electron mobility increase. Again, this is inconsistent with a Schubweg-limited process. The computer simulations show that increasing the higher of the two mobilities should have a much weaker impact on the FF than increasing the lower mobility, and there is no evidence of increased space charge effects.

One of the most important observations that can be made based on Figs. 2 and 3 is that the increase in the FF with respect to increasing electron mobility is comparable in both APFO-3- and APFO-Green 5-based solar cells. The behavior appears to be independent of whether the electron mobilities are higher or lower than the hole mobilities. Although the data is conceptually consistent with a charge separation process, the Onsager-Braun process takes the spatially averaged sum of the mobilities as a parameter, while these results indicate the geometric mean as a more probable parameter since that would always have the same rate of increase with respect to the parametric mobility. In all cases, straight lines offer the best collective fitting of the data compared to the more elaborate shapes required by a process determined by Schubweg- and space-charge-limitations.

Finally, Fig. 4 shows the FF as a function of the experimental parameter molecular weight [Fig. 4(a)], and both the FET and photo-CELIV hole mobility for the APFO3:[60]PCBM blends [Fig. 4(b)]. The data were collected from the 1:4 APFO-3:[60]PCBM blends based on different APFO-3 synthesis batches with different molecular weights. The FET and CELIV electron mobilities for blends of this stoichiometry from the samples underlying Fig. 2 are included as a reference. Photo-CELIV on considerably thicker samples was necessary to obtain the hole extraction currents. However, due to very small extraction currents, incompletely relaxed mobility values are given in Fig. 4. This decreases the difference between the FET and photoCELIV mobilities, and since the samples are of different thicknesses, with higher molecular weights tending to yield thicker films and higher hole mobility, this also influences the slope of the FF versus mobility data due to thermalization effects. In this case the electron mobility is intermediate,
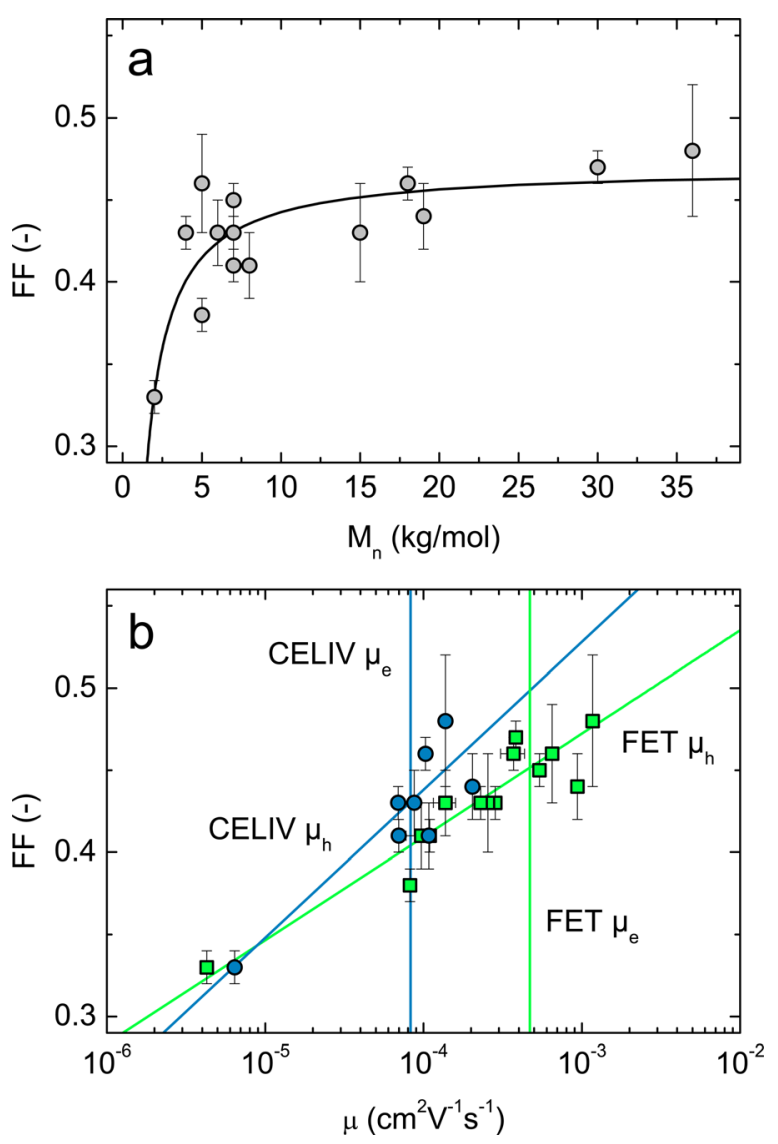

FIG. 4. (Color online) The FF as a function of number average molecular weight fitted assuming a $\mathrm{M}_{\mathrm{n}}^{-1}$ (a) dependence, and (b) mobility. The FET (squares) and photo-CELIV (circles) hole mobilities for 1:4 APFO3:[60]PCBM blends based on different synthesis batches of APFO-3 are shown. The FET and CELIV electron mobilities for this stoichiometry are reproduced from Fig. 2, are constant at approximately $5 \times 10^{-4}$ and $8 \times 10^{-5} \mathrm{~cm}^{2} \mathrm{~V}^{-1} \mathrm{~s}^{-1}$, respectively, and are represented by vertical lines. The FF increases linearly with $\log \left(\mu_{\mathrm{h}}\right)$. 
and there is no clear indication that the equal mobility crossover is of any significance. Whereas the underlying stoichiometry data of Figs. 2(a) and 3(a) confirm the linear dependence of the FF on $\log (\mu)$ due to the excellent fits possible and the general assumption that the electron mobility should have a simple relationship with stoichiometry, it can be argued that the FF versus $\log (\mu)$ of Fig. 4(b) offers a better fit than the FF versus molecular weight of Fig. 4(a). This is due to documented, but unexplained, ${ }^{24}$ synthesis batch differences in the polymer hole mobility in addition to the fundamental molecular weight dependence.

The slope in Fig. 4 is equal to an increase in FF of about 0.064 units per decade of mobility increase, which is considerably less than what is observed for the electron mobilities during the stoichiometry variations. That the polymer mobility has a weaker influence on the FF can be rationalized through dimensionality, especially if we consider that the polymer is quite dilute at only $20 \%$ by weight in the devices upon which Fig. 4 is based. The microscopic (intrachain) mobility is the relevant parameter for the exciton dissociation, and the macroscopic (interchain) mobility that is measured here is but a reflection of it. Microscopic mobility on a single polymer chain is not sensitive to dilution, which is the case for small molecules. This implies that polymer mobility should affect charge-generation dynamics to a lesser extent than the small-molecule mobility where both the microscopic and macroscopic mobility is increased in all three dimensions when the volume fraction is increased.

Another possible reason for the weaker effect of the polymer mobility could be that the increase in delocalization for the negative charges is much stronger with the increasing small molecule (fullerene) fraction, than for the positive charges with increasing molecular weight. A lower exciton binding energy, such as resulting from increased delocalization, also facilitates charge separation just as an increased mobility does.

The empirical relationship between the FF and mobility presented here is consistent with dominating geminate recombination and a FF that is suppressed mainly by the charge separation and not by the actual transport of charge. Furthermore, it implies an exponential relationship between the charge separation and the geometric mean of the carrier velocity. Another observation, which is in agreement with this conclusion, is that the FF seems to follow the same trend well below a FF of 0.25 , which is an inflection point under the Schubweg limitation. This can be seen very clearly in Fig. 2(a).

In fact, it is the microscopic mobility in the vicinity of the charge separation site, ${ }^{26}$ and not the macroscopic mobility (that is typically probed with conventional mobility measurements), which is important for geminate recombination limited devices, and it is thus difficult to say anything a priori about the strength of the dependence of the FF on the macroscopic mobility for a given system. The two are related, however, and as long as the phase structure does not change significantly in the vicinity of the exciton splitting sites at the same time as the mobility changes, the general correlation between FF and mobility shown here should hold for systems limited by geminate recombination. There are plenty of examples where films with very similar mobilities can significantly differ in FF. Indeed, just such a variation is apparent when comparing Figs. 2 and 4, where different solvents have been used to fabricate the active blend layers. Fundamentally, it is a question of the geminate recombination time and morphology. In a system with less-pronounced geminate recombination due to larger (pure) domains and sharper interfaces, lower mobilities can be tolerated. The APFO-3:[60]PCBM blends cast from different solvents is a good example; in general, the macroscopic mobility is independent of the solvent, however, the morphology, and thus the FF, can vary. ${ }^{27}$ A FF primarily limited by charge separation is also consistent with the stronger than expected influence of electrode materials on the FF, since the built-in field has a significant influence.

\section{CONCLUSIONS}

It has been shown that the FF in the polymer:fullerene systems investigated here is limited by the charge separation event and not by the actual transport of the charge to the electrodes, which is in agreement with a dominating geminate recombination, as has been observed with transient absorption. The charge separation is, however, dependent on mobility and thus the FF displays a similar dependency. With this type of limitation, higher mobilities are always beneficial, even if they are very unbalanced, since there are no negative effects from the space charge. In addition, these results highlight the observation that increasing the mobility is an unsatisfactory solution in order to obtain a very high $\mathrm{FF}$, since unrealistically high mobilities would be required. A better option is to engineer the interfaces so that the microscopic charge transport in the appropriate direction can compete more favorably with the geminate recombination. Implications for materials design are that the charge transport should be fast at early times and over the appropriate distances after photo-generation. This will argue for the use of better-ordered or phase-separated materials, on a length scale appropriate for securing charge separation. Enhanced crystallinity or liquid crystallinity may help, and is a known factor of importance in regioregular P3HT-based systems. ${ }^{28}$

\section{ACKNOWLEDGMENTS}

We would like to thank S. Hellström, E. Wang, W. Mammo, and M.R. Andersson for access to the polymers. This work was supported by the Swedish Energy Agency through the Morphoso project and the German Federal Ministry of Education and Research (BMBF) under Contract No. 03SF0331.

\section{APPENDIX: INFORMATION ABOUT THE CALCULATIONS AND COMPUTER SIMULATIONS}

Zeroth order synthetic J-V curves for the Schubweg-limited case were calculated as;

$$
J=\left\{\begin{array}{l}
\frac{\mu \tau}{d^{2}}\left(V_{O C}-V\right), J<C \\
C, J \geq C
\end{array}\right.
$$


TABLE I. Parameters for the numerical modeling.

\begin{tabular}{|c|c|c|c|}
\hline Parameter & Value & Unit & Meaning \\
\hline $\mathrm{d}$ & 100 & $\mathrm{~nm}$ & Thickness of active layer \\
\hline $\mathrm{E}_{\mathrm{G}}$ & 1.4 & $\mathrm{eV}$ & Band gap \\
\hline $\mathrm{N}_{\mathrm{C}}$ & $5 \times 10^{20}$ & $\mathrm{~cm}^{-3}$ & DOS of electron transport level \\
\hline $\mathrm{N}_{\mathrm{V}}$ & $5 \times 10^{20}$ & $\mathrm{~cm}^{-3}$ & DOS of hole transport level \\
\hline$\mu_{\mathrm{e}}$ & $\ldots$ & $\mathrm{cm}^{2} /(\mathrm{Vs})$ & Mobility of electrons \\
\hline$\mu_{\mathrm{h}}$ & $\cdots$ & $\mathrm{cm}^{2} /(\mathrm{Vs})$ & Mobility of holes \\
\hline $\mathrm{e}$ & 3.4 & $\ldots$ & Relative dielectric permittivity \\
\hline G & $3.75 \times 10^{21}$ & $\mathrm{~cm}^{-3} \mathrm{~s}^{-1}$ & Generation rate (spatially homogeneous) \\
\hline $\mathrm{k}_{\mathrm{r}}$ & $6 \times 10^{-11}$ & $\mathrm{~cm}^{3} \mathrm{~s}^{-1}$ & Bimolecular recombination rate constant \\
\hline$\chi_{\mathrm{e}}$ & 4.3 & $\mathrm{eV}$ & Electron affinity \\
\hline$\Psi_{\mathrm{e}}$ & 4.2 & $\mathrm{eV}$ & Work function of electron contact \\
\hline$\Psi_{\mathrm{h}}$ & 5.8 & $\mathrm{eV}$ & Work function of hole contact \\
\hline
\end{tabular}

where $\mathrm{d}$ is the sample thickness, $\mu$ is the mobility, $\tau$ is the carrier lifetime, and $\mathrm{C}$ is the current corresponding to the carrier generation rate. For the Onsager-Braun case, the current was taken as directly proportional to $\mathrm{P}=\mathrm{k}_{\mathrm{d}} /\left(\mathrm{k}_{\mathrm{f}}+\mathrm{k}_{\mathrm{d}}\right)$, with $\mathrm{k}_{\mathrm{f}}$ constant and

$$
k_{d}=\frac{3 \mu e}{4 \pi \varepsilon_{0} \varepsilon_{r} a^{3}} e^{-\Delta E / k_{B} T} J_{1}\left[2 \sqrt{2}(-b)^{1 / 2}\right] /\left[\sqrt{2}(-b)^{1 / 2}\right]
$$

where e is the elementary charge, $\varepsilon_{0}$ is the permittivity of free space, $\varepsilon_{\mathrm{r}}$ is the relative permittivity, a is the charge transfer electron-hole separation distance, $\Delta \mathrm{E}$ is the electron-hole binding energy, $\mathrm{k}_{\mathrm{B}}$ is Boltzmann's constant, $\mathrm{T}$ is the temperature, and $\mathrm{b}=\mathrm{e}^{3} \mathrm{E} /\left(8 \pi \varepsilon_{0} \varepsilon_{\mathrm{r}} \mathrm{k}_{\mathrm{B}}{ }^{2} \mathrm{~T}^{2}\right)$ with $\mathrm{E}$ the electric field. $\mathrm{J}_{1}$ is the Bessel function of order one. The FF was then evaluated in the usual manner. Numerical modeling was carried out with the commercial semiconductor simulation tool, Sentaurus Device ${ }^{29}$, from Synopsys. A finite volume method is used to solve the differential equations for drift and diffusion self-consistently with the Poisson equation. Fermi statistics were used for the electrons and holes. The bulk-heterojunction was mimicked as one effective semiconductor with an electron transport level corresponding to the LUMO of the acceptor and a hole transport level corresponding to the HOMO of the donor phase. It was further characterized by its bandgap, the densities of states, the mobilities of the electrons and holes, and its dielectric permittivity. The effective semiconductor was sandwiched between two (metal) contacts, which were characterized by their work functions and the surface recombination velocities of the electrons and holes. Work functions were chosen such that surface recombination effects were negligible (the work function determines the concentrations of electrons and holes at the semiconductor/metal interface and hence the "selectivity" of that contact). In the bulk, only bimolecular recombination was taken into account. A spatially homogeneous generation rate of "free" charge carriers was used (which corresponds to complete exciton dissociation) and geminate recombination was neglected. All of the simulation parameters are summarized in Table I and have been selected so as to mimic the APFO-3:[60]PCBM blend as closely as possible.

${ }^{1}$ A. M. Ballantyne, L. Chen, J. Dane, T. Hammant, F. M. Braun, M. Heeney, W. Duffy, I. McCulloch, D. D. C. Bradley, and J. Nelson, Adv. Funct. Mater. 18, 2373 (2008).

${ }^{2}$ K. Tajima, Y. Suzuki, and K. Hashimoto, J. Phys. Chem. C 112, 8507 (2008).

${ }^{3}$ P. Vanlaeke, A. Swinnen, I. Haeldermans, G. Vanhoyland, T. Aernouts, D. Cheyns, C. Deibel, J. D'Haen, P. Heremans, J. Poortmans, and J. V. Manca, Solar Energy Materials and Solar Cells 90, 2150-2158 (2006).

${ }^{4}$ D. Gupta, S. Mukhopadhyay, and K. S. Narayan, Sol. Energy Mater. Sol. Cells 94, 1309 (2010).

${ }^{5}$ P. Peumans and S. R. Forrest, Chem. Phys. Lett. 398, 27 (2004).

${ }^{6}$ L. M. Andersson and O. Inganäs, Chem. Phys. 357, 120 (2009).

${ }^{7}$ T. Kirchartz, K. Taretto, and U. Rau, J. Phys. Chem. C 113, 17958 (2009).

${ }^{8}$ C. L. Braun, J. Chem. Phys. 80, 4157 (1984).

${ }^{9}$ L. M. Andersson, F. L. Zhang, and O. Inganas, Appl. Phys. Lett. 91, 071108 (2007).

${ }^{10}$ L. M. Andersson, F. L. Zhang, and O. Inganäs, Appl. Phys. Lett. 89, 142111 (2006).

${ }^{11}$ S. De, T. Pascher, M. Maiti, K. G. Jespersen, T. Kesti, F. L. Zhang, O. Inganäs, A. Yartsev, and V. Sundström, J. Am. Chem. Soc. 129, 8466 (2007).

${ }^{12}$ V. I. Arkhipov, P. Heremans, and H. Bässler, Appl. Phys Lett. 82, 4605 (2003).

${ }^{13}$ J. J. Benson-Smith, L. Goris, K. Vandewal, K. Haenen, J. V. Manca, D. Vanderzande, D. D. C. Bradley, and J. Nelson, Adv. Funct. Mater. 17, 451 (2007).

${ }^{14}$ K. Vandewal, K. Tvingstedt, A. Gadisa, O. Inganas, and J. V. Manca, Nature Mater. 8, 904 (2009).

${ }^{15}$ F. C. Jamieson, T. Agostinelli, H. Azimi, J. Nelson, and J. R. Durrant, J. Phys. Chem. Lett. 1, 3306 (2010).

${ }^{16}$ R. A. Marsh, J. M. Hodgkiss, and R. H. Friend, Adv. Mater. 22, 3672 (2010).

${ }^{17}$ Y. Zhou, K. Tvingstedt, F. L. Zhang, C. X. Du, W. X. Ni, M. R. Andersson, and O. Inganäs, Adv. Funct. Mater. 19, 3293 (2009).

${ }^{18}$ K. Tvingstedt, K. Vandewal, A. Gadisa, F. L. Zhang, J. Manca, and O. Inganas, J. Am. Chem. Soc. 131, 11819 (2009).

${ }^{19} \mathrm{~K}$. Tvingstedt, K. Vandewal, F. L. Zhang, and O. Inganas, J. Phys. Chem. C 114, 21824 (2010).

${ }^{20}$ V. D. Mihailetchi, H. X. Xie, B. de Boer, L. J. A. Koster, and P. W. M. Blom, Adv. Funct. Mater. 16, 699 (2006).

${ }^{21}$ S. Nilsson, A. Bernasik, A. Budkowski, and E. Moons, Macromolecules 40, 8291 (2007).

${ }^{22}$ K. G. Jespersen, F. L. Zhang, A. Gadisa, V. Sundström, A. Yartsev, and O. Inganäs, Org. Electron. 7, 235 (2006).

${ }^{23}$ L. M. Andersson, Org. Electron. 12, 300 (2011).

${ }^{24}$ C. Müller, E. G. Wang, L. M. Andersson, K. Tvingstedt, Y. Zhou, M. R. Andersson, and O. Inganäs, Adv. Funct. Mater. 20, 2124 (2010).

${ }^{25}$ A. M. Goodman and A. Rose, J. Appl. Phys. 42, 2823 (1971).

${ }^{26}$ A. Devizis, K. Meerholz, D. Hertel, and V. Gulbinas, Chem. Phys. Lett. 498, 302 (2010).

${ }^{27}$ F. L. Zhang, K. G. Jespersen, C. Björström, M. Svensson, M. R. Andersson, V. Sundström, K. Magnusson, E. Moons, A. Yartsev, and O. Inganäs, Adv. Funct. Mater. 16, 667 (2006).

${ }^{28}$ S. Shoaee, M. P. Eng, E. Espildora, J. L. Delgado, B. Campo, N. Martin, D. Vanderzande, and J. R. Durrant, Energy Environ. Sci. 3, 971 (2010).

${ }^{29}$ Synopsys, TCAD Sentaurus: Sentaurus Device User Guide, Release D-2010.03; www.synopsys.com (2010). 